\title{
Navigationssystem und Bildgebung, Setup, Wartung und Bedienung
}

\author{
Paul Alfred Grützner, Andreas Wentzensen
}

\section{Zusammenfassung}

Die Bedeutung der computerassistierten Chirurgie wird in den nächsten Jahren noch zunehmen. Schon jetzt haben die intraoperative Navigation, die präoperative Planung und die intraoperative 3D-Bildgebung ihr Potenzial zur Erhöhung der Sicherheit und Präzision bei der Ausführung von chirurgischen Eingriffen bewiesen. Die Eingriffe in den Operationsablauf sind jedoch erheblich. Dies setzt hohe Anforderungen an die Schulung des Personals, aber auch an die Weiterentwicklung der Systeme in Rich- tung verbesserte intraoperative Ergonomie. Gerade in diese Richtung sollten zukünftige Entwicklungen vorangetrieben werden und nicht nur in die reine Machbarkeit von Vorgehensweisen. Die Navigation wird in $\mathrm{Zu}-$ kunft auch einen deutlichen Stellenwert in der Aus- und Weiterbildung von Chirurgen erlangen. Diese Verfahren sind längst nicht mehr schöne Spielereien für Computerfreaks, sondern gehören in vielen Operationssälen bereits zur Standardausrüstung, wie Bildverstärker und Endoskopieturm.

\section{Einleitung}

In einer zunehmenden Zahl von Kliniken in Deutschland und Europa kommen Navigationssysteme zum Einsatz. In der überwiegenden Zahl der Fälle werden diese Systeme bei der Implantation von Kunstgelenken an der Hüfte und am Kniegelenk eingesetzt. Endoprothetik ist in der Regel gut planbar. Es kann daher auch in der Routineversorgung gewährleitstet werden, dass geschulte Teams zur Verfügung stehen die den klinischen Einsatz unterstützen. Im Bereich der Knieendoprothetik kristallisiert sich anhand der Studiendaten heraus, dass in Zukunft die Navigation einen Standard darstellen wird.

Völlig andere Voraussetzungen, bezüglich des intraoperativen Einsatzes rechnergestützter Verfahren liegen beim Trauma vor. Aufgrund der begrenzten Planungszeit beim Frischverletzten, der einge-

OP-JOURNAL 2005; 21: 46-50

(c) Georg Thieme Verlag KG Stuttgart · New York schränkten Verfügbarkeit von geschulten Teams, vor allem im Nachtdienst und den systembedingten Defiziten bei der Reposition der Frakturen und auch dem Verfolgen mehrerer Frakturfragmente, ist man im Bereich der Extremitäten von einem Einsatz in der klinischen Routine noch weit entfernt. In topographisch schwierigen Regionen am Becken und an der Wirbelsäule kann die Navigation in der Hand eines geübten Operateurs zu einer Verbesserung der Qualität führen.

Verbesserungen in der Platzierung von Implantaten durch computerassistierte Verfahren, die Möglichkeit einer sofortigen Ergebniskontrolle und eine reduzierte Strahlenbelastung für Arzt und Patient sind die ideale Ergänzung zu minimalinvasiven Techniken.

In der Unfallchirurgie und Orthopädie wird in Zukunft die Verbesserung der intraoperativen Bildgebung von großer Bedeutung sein. Mit der Einführung dreidimensionaler Röntgenbildverstärker wird es zu einer Verbesserung der Darstellung, gerade bei Gelenkfrakturen, bei Wirbelsäulen- und Beckeneingriffen im Opera- tionssaal kommen. Diese Geräte können direkt an ein Navigationssystem angebunden werden. Die Entwicklung in diesen Bereich ist rasant und wird zu einem langsamen, aber nachhaltigen Durchsetzen der Verfahren in den Bereichen führen in denen ein Nutzen für die Patienten erzielt werden kann.

Diese Technologisierung im OP stellt aber extreme Anforderungen, sowohl von Seiten der OP-Pflege, als auch auf ärztlicher Seite.

Ein erfolgreicher Einsatz der Navigation und auch der dreidimensionalen Bildgebung kann nur durch eine intensive Schulung der Anwender erfolgen. Fehlschläge beim navigierten Operieren sind in den meisten Fällen auf Anwendungsfehler und Fehlinterpretation durch den Chirurgen zurückzuführen.

Die Industrie verspricht eine einfache Anwendung der Systeme. Wenig ist bisher über die Implementierung dieser Systeme in die klinische Routine und die Standardisierung der Vorgehensweisen berichtet worden.

\section{Intraoperative 3D-Bildgebung mit mobilen Bildverstärkern}

\section{Funktionsprinzip}

Die intraoperative 3D-Bildaquisition mithilfe von Röntgenbildverstärkern beruht auf einer 2D-Datenaufnahme in definierten Winkelabständen. Für diese Datenaufnahme rotiert der Bildverstärker in festen Winkelabständen um die entsprechende Region am Patienten. Aus den einzelnen Röntgenprojektionen errechnet der Computer im Monitorwagen des C-Bogens einen isotropen 3D-Datenwürfel. Da der 3D-Datenwürfel aus den einzelnen Röntgenprojektionen errechnet wird, müssen einige Grundregeln beachtet werden. Während der Bildaufnahme darf sich der Patient in Relation zum Bildverstärker nicht bewegen. Hierbei ist es wichtig zu beachten, dass es auch zu 
keiner Kollision mit den Lagerungsmitteln oder Patienten kommt. Es muss außerdem gewährleistet sein, dass möglichst wenig patientenfremde röntgendichte Strukturen im Strahlengang vorhanden sind. Diese Strukturen, wie z.B. Tischverstrebungen aus Metall, Kontraststreifen in Tupfermaterialien oder sonstiges Metall, führen zu erheblichen Artefakten. Der Patient muss daher auf röntgendurchlässigen Operationstischen (Carbonmaterial) gelagert sein.

\section{Lagerung}

Die Patientenlagerung orientiert sich selbst an operativen Kriterien. Aufgrund der technischen Gegebenheiten des Bildverstärkersystems müssen unter Umständen Kompromisse gemacht werden. Z.B. bei Frakturen am Hüftgelenk kann es erforderlich werden, den Patienten möglichst in der Tischmitte zu lagern um ein Herumschwenken des Bildverstärkers um die zu untersuchende Struktur gewährleisten zu können.

Es gibt mittlerweile ein großes Angebot an Carbonlagerungshilfen, so stehen komplette Carbontischsysteme mit durchgängigen Platten zur Verfügung, die eine artefaktfreie Röntgenuntersuchung über einen großen Bereich ermöglichen. Dies ist besonders bei Eingriffen am Becken äußerst hilfreich. Bei vielen Indikationen können auch Carbonanstecksysteme an vorhandene Tischsysteme verwendet werden, die Anstrecksysteme gibt es für den Kopf-Hals-Bereich, für die untere Extremität und die obere Extremität (Abb.1).

Bei Operationen im Rumpfbereich hat sich bei den durchgängigen Carbontischen die zusätzliche Verwendung einer Vacuummatratze zur Lagerung als äußerst komfortabel gezeigt. Im Gegensatz zu den Vakuummatratzen aus dem Rettungsdienst sind bei den OP-Vakuummatratzen das Füllmaterial und die Außenhaut artefaktfrei durchstrahlbar. Die Vakuummatratze kann beliebig anmodelliert werden, ist in evakuiertem Zustand formtreu, steif und kippstabil. Hierdurch kann auf den Einsatz von Symphysenund Thoraxstützen weit gehend verzichtet werden. Der Patient liegt gut wärmeisoliert. Es ist jedoch darauf $\mathrm{zu}$ achten, dass beim Einsatz der Vakuummatratze keine feuchte Kammer entsteht, eine zusätzliche Verwendung eines Baumwolltuches ist hierbei anzuraten.

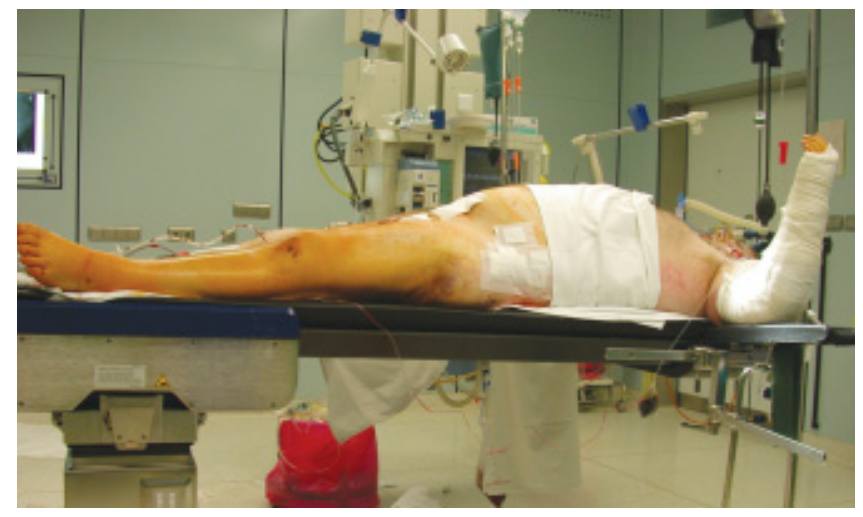

Abb. 1 Lagerung auf einer durchgängigen Carbonplatte mit artefaktfreier Durchleuchtungsmöglichkeit des gesamten Rumpfes. (Gewicht des Patienten: $165 \mathrm{kp}$ ).

\section{Hygiene und sterile Abdeckung}

Grundsätzlich ist beim intraoperativen Einsatz eines Bildverstärkers auf die erforderlichen Hygienemaßnahmen streng zu achten. In der Regel gilt die OP-Tischunterseite generell als unsteriler Bereich. Durch die erforderliche Orbitalbewegung bei der dreidimensionalen Bildaufnahme, bei der die Bildquelle unter dem Tisch durchschwenkt, ist davon auszugehen, dass die Bildquelle unsteril ist. Es ist zu empfehlen, sowohl den Röntgenbildverstärker mit sterilen Abdeckfolien zu versehen, als auch zusätzliche sterile Tücher im Operationsfeld $\mathrm{zu}$ verwenden. Im Zweifelsfall sollte nach Herausfahren des Bildverstärkers das Operationsfeld neu steril eingedeckt und während der Bildaufnahme kann ggf. der Operationssitus zusätzlich steril abgedeckt werden (Abb. 2).

Bei der Orbitalbewegung besteht das Problem, das seitliche herunterhängende Abdeckungstücher vom C-Bogen aufgeladen werden und somit vom unsterilen Bereich in das Operationssitus geschoben werden können. Es empfiehlt sich daher den Operationssitus steril abzukleben und mit einem sterilen Tuch zu schützen. Als praktikabel hat sich, um das Aufladen von Tüchern zu vermeiden, eine sogenannte Tunnelabdeckung erwiesen, durch sterile Abdeckungstücher werden die seitlich herunterhängenden Tücher vom Tischende vorsichtig aufgenommen und mit Klebe-Taps fixiert. Besonders einfach kann eine Tunnelabdeckung durch einen sterilen Instrumentiertischüberzug vom Tischende aus über den Tisch geschoben werden.

Lagerung bei Intraoperativer 3D-Bildgebung:

— Artefaktfrei röntgendurchlässige Lagerungsfläche aus Carbon

- Ausschalten von Patientenbewegung während der Bildaufnahme

- Respektierung der hygienischen Anforderungen

\section{OP-Ablauf}

Nach der Lagerung des Patienten ist es wichtig, vor dem sterilen Abdecken die Durchleuchtungsmöglichkeiten zu überprüfen. Der Bildverstärker und Monitorwagen wird gegenüber dem Operateur

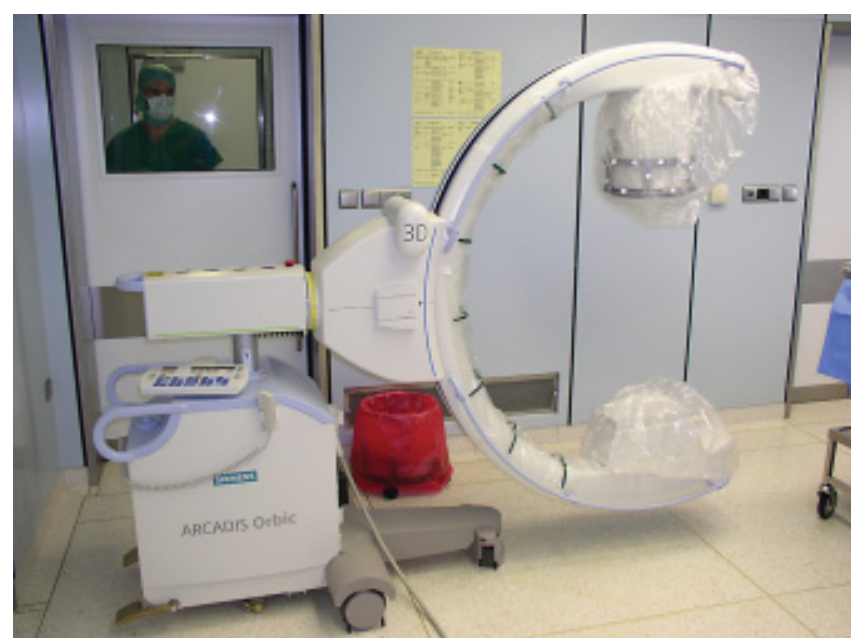

Abb. 2 3D-Bildverstärker mit sterilem Bezug. 
bzw. am Fuß- oder Kopfende so gestellt, dass der Operateur unter ergonomischen Gesichtspunkten eine optimale Sicht auf das System erhält.

Der Einsatz der 3D-Bildgebung intraoperativ hängt von der individuellen intraoperativen Situation ab. Der Bildverstärker kann aus rein diagnostischen Zwecken zu einem besseren Verständnis der Situation eingesetzt werden oder er kann eingesetzt werden, um Daten für ein navigiertes Vorgehen aufzunehmen (siehe unten). In Zwischenschritten nach Reposition und temporärer Fixation kann eine Kontrolle erfolgen. In den meisten Fällen wird aber eine Abschlusskontrolle der Osteosynthesen erfolgen. Diese Abschlussuntersuchung wird dann in vielen Fällen eine postoperative CT-Untersuchung ersetzen. Der 3D-Bildverstärker ist in der Bedienung der eines konventionellen 2D-Bildverstärkers sehr ähnlich und kann auch als solcher eingesetzt werden. Beachtet werden muss die Möglichkeit einer freien Rotation des Bildverstärkers um die operierte Struktur. Der Bildverstärker wird daher vor der Aufnahme der dreidimensionalen Daten entsprechend eingerichtet. Als Hilfsmittel stehen hierzu Laserlichtvisiere und optische Kontrollmöglichkeiten zur Verfügung.

\section{Bildbearbeitung und Dokumentation}

Die intraoperative Aufarbeitung der aufgenommenen 3D-Daten erfolgt unter speziellen Bedingungen. Der Patient liegt in Narkose, diese Narkose kann durch den besonderen Aufwand der 3D-Bildgebung verlängert werden. Der Operateur trägt die Verantwortung für die intraoperative Bildgebung und die Interpretation der Bilddaten. Er muss daher in einer möglichst kurzen Zeit die Information möglichst optimal auswerten, denn nur jetzt kann in der gleichen Narkose auf die Ergebnisse der 3D-Bildgebung reagiert werden. Eine effiziente Nutzung dieser Information ist daher nur beim Einhalten standardisierter Arbeitsabläufe gewährleistet. Die Information muss in möglichst wenigen Arbeitsschritten mit einer hohen Treffsicherheit aus den Bildern gezogen werden. Es muss gewährleistet sein, dass eine Person, die in der Beurteilung dieser Bilder geschult ist, im Operationssaal verfügbar ist. Die Ergonomie dieser Systeme erlaubt es noch nicht, die Bildbearbeitung vom Operationstisch steril durchzuführen. Durch den Operateur müssen die mit der Bildgebung verbundenen Fragestellungen präzise formuliert

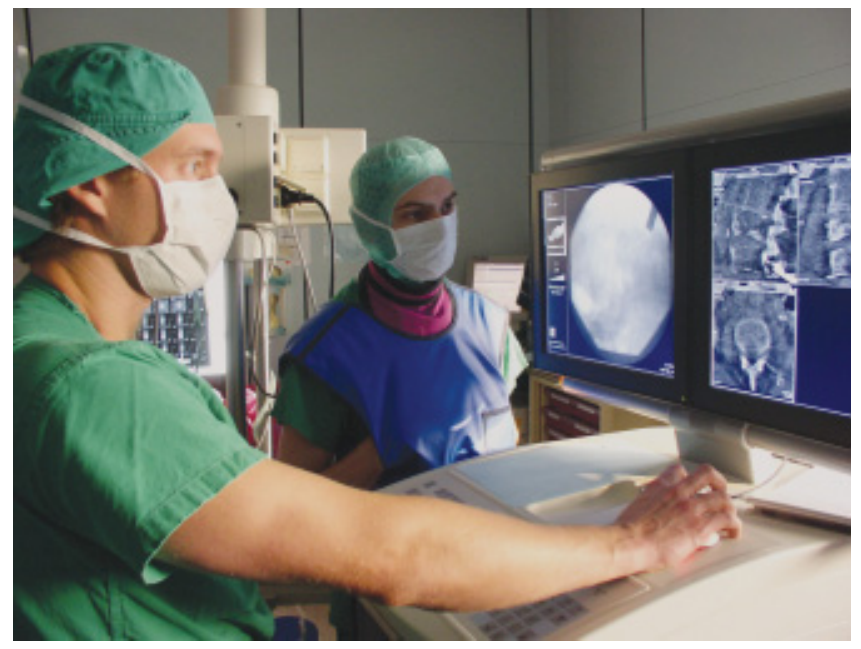

Abb. 3 Intraoperative Bildbearbeitung am Monitorwagen des 3D-Bildverstärkers.

sein. Es muss klar ersichtlich sein, welche Konsequenzen er ggf. aus diesen Informationen ziehen wird. Grundsätzlich kann die Bildbearbeitung durch den Operateur selbst, durch geschulte Assistenten oder auch durch geschultes OP-Personal erfolgen (Abb. 3).

Bei der Bearbeitung der Bilddaten ist es extrem wichtig, sich an den Grundprinzipien der Interpretation bei Schnittbilddiagnostik zu orientieren. Hierzu gehört eine Einstellung optimaler Helligkeitsund Kontrastwerte, das Wählen einer korrekten Schichtdicke für die Interpretation der Bilder und Artefaktreduktion, und vor allem die Darstellung der Bilddaten durch Einstellen der standardisierten, von der CT- und MR-Diagnostik bekannten Ebenen. Die Besonderheit des Datenkubus aus der dreidimensionalen Bildgebung liegt darin, dass der Patient nicht standardisiert zum C-Bogen gelagert werden kann. Außerdem fehlen aufgrund des limitierten Scanvolumens, die teilweise bekannten Landmarken, was vor allem bei Anwendungen im Beckenbereich die Schwierigkeit der Bildinterpretation erhöht.

Für die intraoperative 3D-Bildgebung gilt, wie für jedes andere Röntgengerät auch, die Röntgenverordnung. Dementsprechend müssen die intraoperativ erhobenen Daten, gemäß den gesetzlichen Bestimmungen archiviert und die intraoperativen Durchleuchtungszeiten dokumentiert werden. Auch hier trägt die Verantwortung der Operateur. Idealerweise erfolgt die Anbindung des Gerätes an die PACS-Struktur der Klinik.

Die intraoperative Bildgebung stellt hohe Anforderungen an die Schulung des OP-Personals. Die Bildbearbeitung und Dokumentation orientiert sich an CT-Standards.

\section{Navigation}

Der Einsatz von Navigationssystemen in der Unfallchirurgie und Orthopädie findet eine zunehmende Verbreitung. Dieser Fortschritt versucht dem Anspruch der Verbesserung der Patientenversorgung gerecht zu werden. Intraoperative Navigation bedeutet die Verknüpfung der Position chirurgischer Instrumente mit patientenbezogenen Informationen. Der Einsatz der Navigation bedeutet einen zusätzlichen intraoperativen Aufwand. Am heute weitesten verbreitet sind Navigationssysteme mit einer optoelektronischen Verfolgung der Instrumente und der Patientenkoordinaten. Das Navigationssystem besteht also aus einem Computer- und Monitorwagen sowie aus einer Kamera. Zur Navigation sind spezielle Instrumente, die mit Markern versehen sind, erforderlich, es setzen sich hier zunehmend kabellose Marker durch, die entweder mit einer eigenen Stromversorgung Impulse an die Kamera senden (aktive Systeme) oder über spezielle Phonien von der Kamera ausgesendete Lichtimpulse reflektieren (passive Systeme) (Abb.4).

Diese Instrumente bedürfen im Umgang einer besonderen Sorgfalt. Die Navigationspräzision hängt letztendlich auch von der Präzision dieser Instrumente ab. Bis auf die Markerkugeln der passiven Systeme und die Batterien der aktiven Systeme sind sämtliche Instrumente dampfsterilisierbar. Bei dem Reinigungsund Sterilisationsprozess muss darauf geachtet werden, dass es zu keiner mechanischen Beanspruchung der Instrumente kommt. Bereits leichte Verbiegungen 


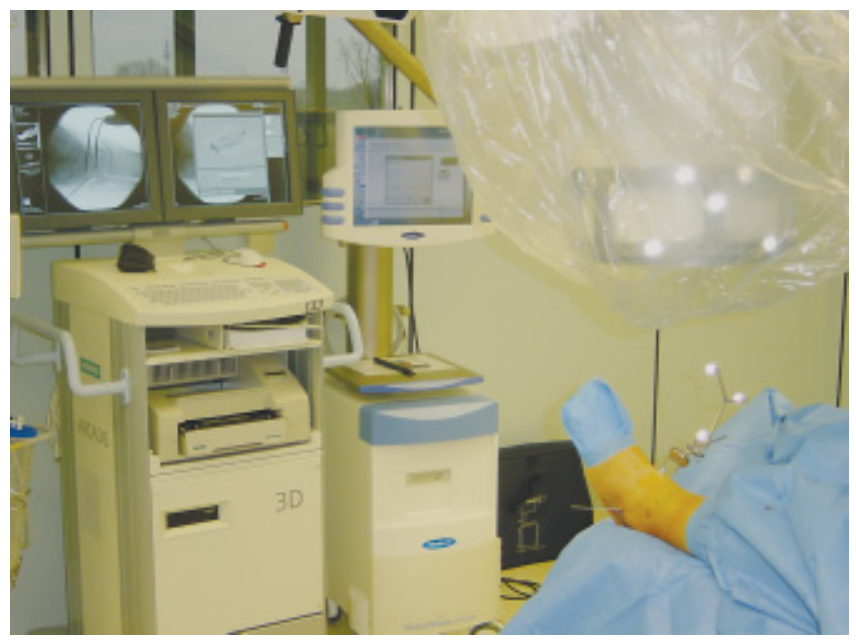

Abb. 4 Monitorwagen des Bildverstärkers, Monitor des Navigationssystems, Markerring am Bildverstärker, Referenzkörper am Patienten.

können fatale Folgen auf die Navigationspräzision haben. Der intraoperative Umgang mit den navigierbaren Instrumenten bedeutet zunächst einen deutlichen Zusatzaufwand. Die Instrumente müssen vor dem eigentlichen Eingriff aufgerüstet werden. Die Markerkugeln werden an den passiven Instrumenten befestigt. Die Markerkugeln sind Einwegmaterial, aus Gründen der Präzision sind die $\mathrm{Ku}-$ geln auch während des Eingriffs vor Verschmutzungen $\mathrm{zu}$ schützen. Falls eine Reinigung von Blutspritzern erforderlich wird, erfolgt diese vorsichtig mit angefeuchteten Kompressen. Vor dem operativen Eingriff ist es zwingend erforderlich, die Präzision dieser Instrumente zu überprüfen. In der Regel erfolgt dies über vom System vorgegebene Schritte im Operationsablauf.

Die Navigation selbst erfolgt dann intraoperativ, entweder in präoperativ aufgenommenen Bilddaten (CT-basierte Navigation), in intraoperativ aufgenommenen Bilddaten (2D oder 3D-bildwandlerverstärkte Navigation) oder durch Registrierung von Landmarken am Patienten direkt. Alle drei Arten der Navigation haben systemimmanente Vor- und Nachteile und ihr spezifisches Indikationsspektrum. Auch hier gilt, dass nur durch festgelegte Protokolle und geschultes Personal ein reibungsfreier Ablauf zu gewährleisten ist (Abb.5).

Das intraoperative Setup des Navigationssystems orientiert sich an ergonomischen Begebenheiten, aber auch an der Funktionsweise des Systems. Es muss gewährleistet sein, dass der Operateur eine freie Sicht zum Computermonitor erhält. Auf der anderen Seite ist es erforderlich, dass die Kamera des Navigationssystems so platziert ist, dass eine freie Sichtlinie zum Operationssitus vorhanden ist. Nur durch die Einhaltung standardisierter Abläufe und die Anwendung der Systeme in der klinischen Routine ist ein entsprechendes Training des OP-Personals zu erwarten. Es bewährt sich daher die Navigation bei bestimmten Eingriffen grundsätzlich einzusetzen, um in schwierigen Situationen die erforderliche Routine aufzuweisen.

Die Bedienung des Navigationssystems intraoperativ erfolgt entweder aus dem sterilen Bereich über einen „touch screen", über Fußschalter, oder aber durch einen zusätzlichen „unsterilen“ Assistenten am Navigationssystem selbst. Die klinische Routine zeigt hier, dass bei standardisierten Eingriffen und bei einem durchdachten Workflow des Computersystems eine sterile Bedienung des Navigationssystems ohne weiteres möglich ist. Bei komplexen Eingriffen, wie z.B. Osteosynthesen am Becken hat es sich bewährt, dass die Bedienung des Systems ein Assistent vornimmt.

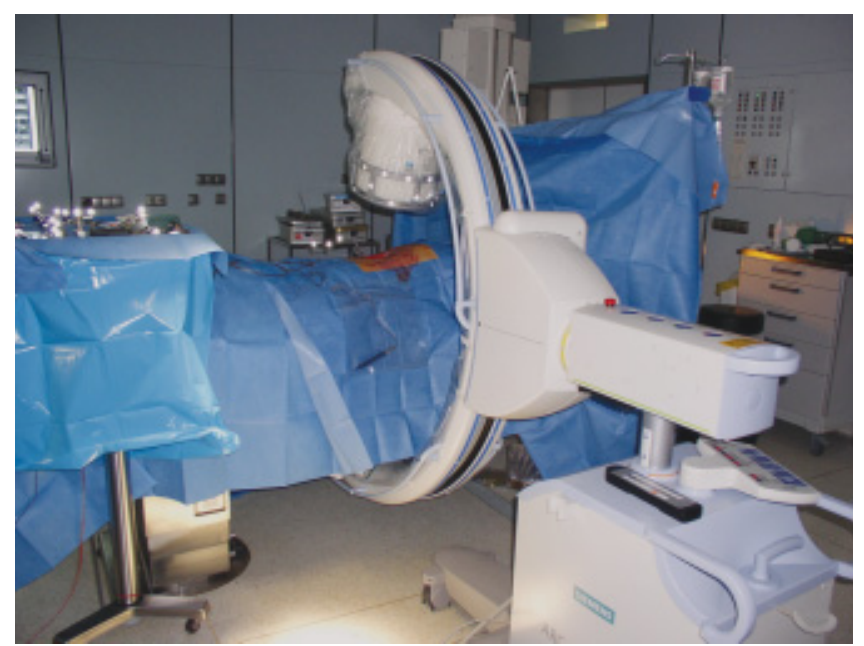

Die drei häufigsten Fehlerursachen bei der Navigation sind die nicht korrekte Registrierung der Patientenanatomie, Probleme bei der Befestigung des Referenzierungskörpers am Patienten und das Verbiegen von Instrumenten während dem Eingriff.

Die Registrierung der Patientenanatomie kann entweder manuelle, wie bei der CTbasierten Navigation erfolgen, hierzu ist es durch Abnehmen von Landmarken erforderlich, den präoperativen CT-Datensatz der Patientenanatomie anzugleichen. Die entscheidenden Vorarbeiten erfolgen hier präoperativ. Der CT-Datensatzes wird präoperativ segmentiert und Landmarken werden geplant, die intraoperativ gefunden werden müssen. Nur durch eine korrekte Digitalisierung der Landmarken am Patienten und somit einer korrekten Registrierung der Patientenanatomie ist eine erfolgreiche Navigation möglich. Dies gilt auch für die bildfreie Navigation z.B. im Bereich der Knieendoprothetik. Diesem Schritt muss daher besondere Sorgfalt gelten. Bei der automatischen Registrierung der Bilddaten durch intraoperative Bildgebung mit dem 2D- oder 3D-Bildverstärker kann es zu Fehlern durch Bewegungen des Patienten während der Bildaufnahme, durch Artefaktbildungen oder ein nicht korrektes Aufrüsten des Navigationssystems kommen. Aus diesem Grund muss nach jeder Registrierung, gleich ob manuell oder automatisch, eine Überprüfung der Genauigkeit erfolgen. Dies kann nur durch den verantwortlichen Operateur selbst erfolgen, er muss Überprüfen, ob die Position eines navigierten Instrumentes vom Navigationssystem exakt an der anatomisch zugeordneten Stelle angezeigt wird. Diese Überprüfung muss an verschiedenen Stellen im Daten-

Abb. 5 Scan mit dem 3D-Bildverstärker zur Navigation an der Wirbelsäule. Der Bildverstärker ist steril bezogen. Gut sind die reflektierenden Markerkugeln am Bildverstärker, am Patienten und an den Instrumenten auf dem Instrumentiertisch zu erkennen. 


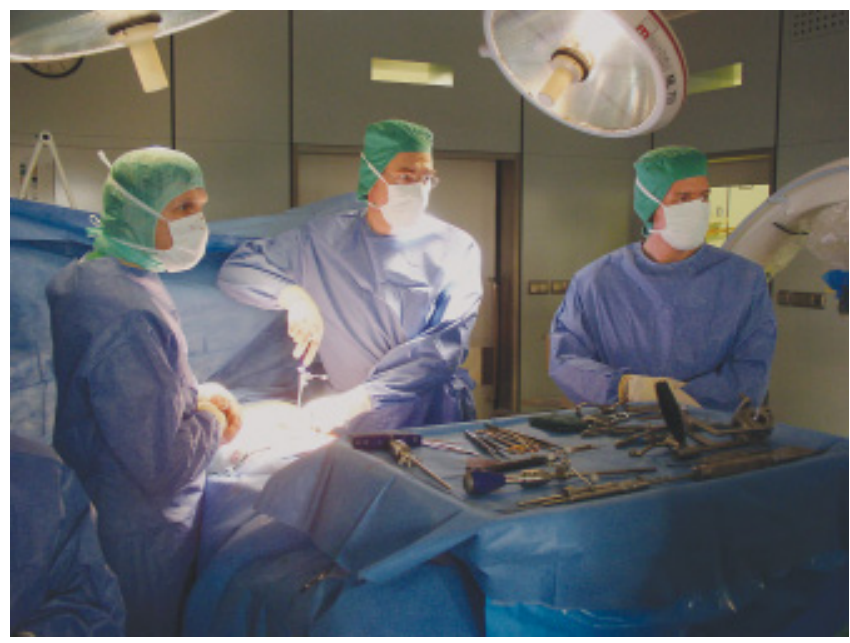

Abb. 6 Ergonomie der navigierten Pedikelinstrumentierung. Der Bildverstärker ist aus dem OP-Feld entfernt. Die Position der Pedikelale kann auf dem Monitor des Navigationssystems verfolgt werden.

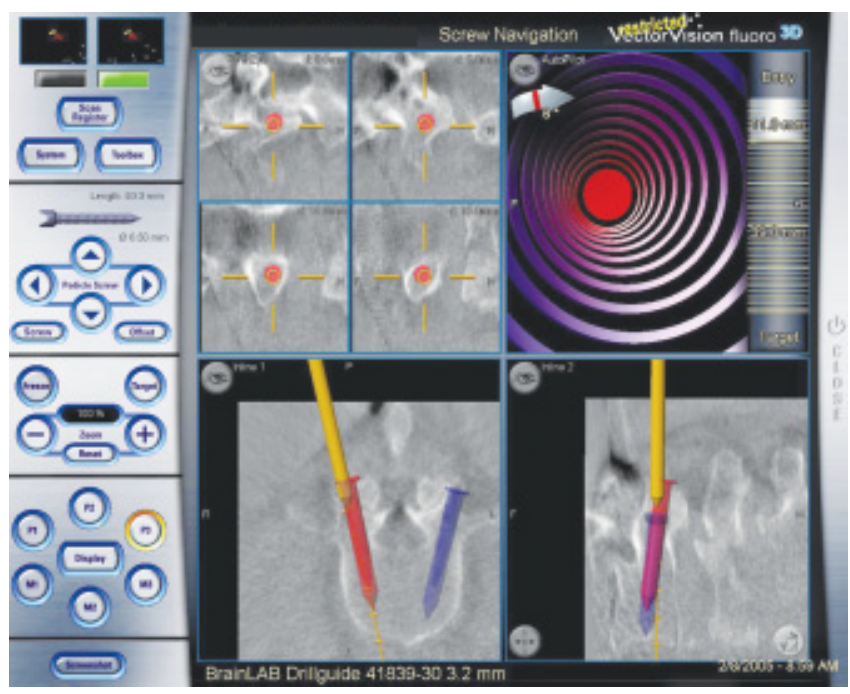

Abb. 7 Korrespondierende Anzeige auf dem Monitor des Navigationssystems.

satz erfolgen, um auch die Möglichkeit einer Verdrehung oder Verkippung des Datensatzes auszuschließen (Abb.6,7).

Die Befestigung des Referenzierungskörpers am Patient muss rotations- und biegungsstabil erfolgen. Die Fixierung muss den Anforderungen des operativen Eingriffs gerecht werden. In der Hüftendoprothetik muss sie den erheblichen $\mathrm{Vi}$ brationen während des Fräsvorganges und den erheblichen Krafteinwirkungen bei der Präparation des Femurschaftes standhalten. Auch bei Rotation z.B. des Femurs bei der navigierten Marknage- lung treten erhebliche Kräfte auf die Fixierung des Referenzierungskörpers auf. Die Befestigung der Referenzierungskörpers muss vom Operateur bei den entscheidenden Operationsschritten überprüft werden.

Ein wichtiger Aspekt ist die Möglichkeit der Verbiegung von Bohrern, Sägeblättern und Implantaten. Es liegt auf der Hand, je verwindungsstabiler das Instrument ist und je exakter die Verbindung mit dem Referenzierungskörper desto präziser ist das Instrument. Es ist daher nicht möglich, Kirschner-Drähte oder dünne Bohrer ohne entsprechende Führungshülsen, die selbst navigiert sein müssen, für die Navigation zu verwenden.

Gerade in kritischen Regionen an der HWS oder am Becken sollte daher im Zweifelsfall die Lage des Instruments mit dem 2D-Bildverstärker während dem navigierten Eingriff überprüft werden.

\section{Dokumentation}

Bei den heute verfügbaren modernen $\mathrm{Na}$ vigationssystemen erfolgt im Hintergrund, ohne für den Operateur ersichtlich, die Dokumentation sämtlicher navigiert durchgeführter operativer Schritte einschließlich Registrierung der Patientenanatomie, Planung von Landmarken und der navigierten Handlung selbst. Diese Daten sollten mit der gleichen Sensibilität wie Röntgendaten behandelt werden. Sie gehören zum Report des Patienten, die Navigation muss in den Operationsbericht mit aufgenommen werden.

\section{Schlußfolgerung}

Computerassistierte Verfahren lassen sich im OP nur dann kosteneffizient, zeitsparend und zum Nutzen für den Patienten einsetzen, wenn sich alle Beteiligten intensiv mit Bedienung und Pflege der Systeme aber auch der Dokumentation der Befunde auseinandersetzen. Nur durch intensive Schulungsmaßnahmen kann das Potential der Systeme ausgenutzt und Fehler vermieden werden.

\section{Dr. med. Paul Alfred Grützner}

Oberarzt

Prof. Dr. med. Andreas Wentzensen

Ärztlicher Direktor

BG-Unfallklinik Ludwigshafen

Klinik für Unfall- und

Wiederherstellungschirurgie an der

Universität Heidelberg

Ludwig-Guttmann-Str. 13

D-67071 Ludwigshafen 